\title{
2 Der neue Rechtsrahmen
}

Die Datenschutz-Grundverordnung vom 27. April 2016 wird ab dem 25. Mai 2018 in allen Mitgliedstaaten unmittelbar gelten und in diesen das Datenschutzrecht auf eine neue Grundlage stellen. Die Datenschutz-Grundverordnung wird dann Teil der jeweiligen nationalen Rechtsordnung sein, ohne diese aber formell zu verändern.

\subsection{Datenschutz-Grundverordnung}

Die Verordnung (EU) 2016/679 des Europäischen Parlaments und des Rates vom 27. April 2016 zum Schutz natürlicher Personen bei der Verarbeitung personenbezogener Daten, zum freien Datenverkehr und zur Aufhebung der Richtlinie 95/46/EG (Datenschutz-Grundverordnung)4 gilt vom 25. Mai 2018 an mit all ihren Regelungen in allen Mitgliedstaaten unmittelbar. Sie bestimmt vorrangig das künftige Datenschutzrecht in Europa. Dieses wird aber nicht allein durch die Union geprägt. Vielmehr wird sie in vielen Bereichen und Aspekten ergänzt durch Datenschutzregelungen der Mitgliedstaaten. Dadurch entsteht im Datenschutz eine Ko-Regulierung durch Union und Mitgliedstaaten. Erst das komplizierte Zusammenwirken von Unionsrecht und Recht der Mitglied-

4 EU ABI. L 119 vom 4.5.2016, 1. 
staaten bewirkt das zukünftige Europäische Datenschutzrecht. ${ }^{5}$ Dies widerspricht zwar vielen Erwartungen und Wünschen nach einem einfachen und einheitlichen Datenschutzrecht in der Union, ${ }^{6}$ ist aber - nüchtern betrachtet das Ergebnis des politischen Prozesses, der die Datenschutz-Crundverordnung hervorgebracht hat. ${ }^{7}$

Die Verordnung verfolgt drei Zielsetzungen: ${ }^{8}$ Zum einen will sie den Datenschutz angesichts der Herausforderungen der technischen Entwicklung modernisieren und den Schutz der Grundrechte verbessern. ${ }^{9}$ Zum anderen will sie das Datenschutzrecht unionsweit harmonisieren und einen soliden, „kohärenten und durchsetzbaren Rechtsrahmen im Bereich des Datenschutzes in der Union" schaffen. ${ }^{10}$ Schließlich will sie einheitliche Vorgaben für gleiche wirtschaftliche Bedingungen in der Union bieten und damit den Binnenmarkt stärken. ${ }^{11}$

Die Datenschutz-Grundverordnung regelt das von ihr erfasste Datenschutzrecht in elf Kapiteln mit 99 Artikeln. Kapitel I enthält in den Art. 1 bis 4 allgemeine Bestimmungen, Kapitel II nennt in Art. 5 bis 10 die allgemeinen Grundsätze des Datenschutzrechts, Kapitel III regelt in den Art. 11 bis 23 die Rechte der betroffenen Person, Kapitel IV bestimmt in Art. 24 bis 43 die Pflichten des Verantwortlichen und des Auftragsverarbeiters und Kapitel V beschreibt in Art. 44 bis 50 die Vorgaben zur Übermittlung personenbezogener Daten in Drittländer oder an internationale Organisationen. Nimmt man zu diesen Vorschriften noch die Regelung zur Haftung der Datenverarbeiter in Art. 82 hinzu, so regelt die Datenschutz-Grundverordnung das materielle Datenschutzrecht in nur 51 Artikeln. Die verbleibenden 48 Artikel beantworten überwiegend organisatorische Fragen der Datenschutzaufsicht und der Regelungskompetenzen und betreffen weitere formelle Themen. In Kapitel VI regelt die Verordnung in den Art. 51 bis 59 die Aufgaben und Befugnisse der unabhängigen Aufsichtsbehörden, in Kapitel VII enthält sie in Art. 60 bis 76 Vorgaben zur Zusammenarbeit und zur Kohärenz der Entscheidungen der Aufsichtsbehörden und in Kapitel VIII regelt sie in Art. 77 bis 84 Rechtsbehelfe und Sanktionen. Kapitel IX enthält in Art. 85 bis 91 Vorschriften für besondere Datenverarbeitungssituationen, Kapitel X gibt in Art. 92 und 93 Vorgaben für delegierte Rechtsakte und Durchführungsrechtsakte und Kapitel XI enthält in Art. 94 bis 99 Schlussbestimmungen.

5 S. näher Roßnagel, in: ders. 2018, §1 Rn. $1 \mathrm{ff}$.

6 Insbesondere von am Entstehungsprozess Beteiligten - s. z.B. Selmayr/Ehmann, in: Ehmann/Selmayr 2017, Einleitung Rn. 3; Albrecht, CR 2016, 97ff.

7 S. zu diesem ausführlich Roßnagel, in: ders. 2018, \1 Rn. $15 \mathrm{ff}$.

8 Diese werden weitgehend verfehlt - s. Roßnagel, in: ders. 2017, $§ 1$ Rn. $27 f f$.

9 S. hierzu Erwägungsgrund 1, 2, 3a und 5 DSGVO.

10 S. hierzu Erwägungsgrund 3 und 6 DSGVO.

11 S. Erwägungsgrund 4 und 8 DSGVO. 
Die neue Verordnung orientiert sich in weiten Teilen weiterhin an den alten Zielen und Grundsätzen der Datenschutzrichtlinie $95 / 46 / \mathrm{EG}^{12}$ von $1995 .{ }^{13} \mathrm{Sie}$ übernimmt unter anderem in Art. 2 und 3 DSGVO weitgehend die Regelungen zum sachlichen und räumlichen Anwendungsbereich, in Art. 5 DSGVO nahezu unverändert die Crundsätze der Datenverarbeitung, in Art. 6 Abs. 1 DSGVO wörtlich die Voraussetzungen für die Zulässigkeit der Datenverarbeitung und in Art. 9 DSGVO grundsätzlich die Regelungen zu besonderen Kategorien personenbezogener Daten. Hinsichtlich der Rechte der betroffenen Person orientiert sie sich in den Art. 12 bis 23 DSGVO ebenfalls stark an der Richtlinie. In Art. 26 und 27 DSGVO greift die Verordnung grundsätzlich auf die Vorgaben der Richtlinie zur Auftragsverarbeitung zurück. In Art. 32 DSGVO übernimmt sie weitgehend die Anforderungen an die Datensicherheit, in Art. 44 bis 50 DSGVO konzeptionell die Grundsätze zur Datenübermittlung in Drittländer und in Art. 51 bis 59 DSGVO die Konzeption der Stellung und Aufgaben der Aufsichtsbehörden. Diese Regelungen werden in der Verordnung präzisiert, neugestaltet oder erweitert, aber konzeptionell nicht weiterentwickelt.

Innovativ ist dagegen in Art. 3 Abs. 2 DSGVO die Ausweitung des räumlichen Anwendungsbereichs durch das Marktortprinzip. Danach ist die Verordnung auch anwendbar, wenn ein Datenverarbeiter personenbezogene Daten von Personen verarbeitet, die sich in der Union aufhalten. Dies gilt allerdings nur, wenn der Verarbeiter entweder der betroffenen Person Waren oder Dienstleistungen anbietet oder die Datenverarbeitung der Beobachtung ihres Verhaltens in der Europäischen Union dient. ${ }^{14}$ Bisher unbekannt ist das Recht für betroffene Personen in Art. 2o DSGVO, ihre Daten, die sie einem Verantwortlichen bereitgestellt haben, auf einen anderen Datenverarbeiter zu übertragen. Innovativ sind auch die Anforderungen an den Datenschutz durch Systemgestaltung und Voreinstellungen in Art. 25 DSGVO und die Datenschutz-Folgenabschätzung in Art. 35 DSGVO. Die engere Zusammenarbeit der Aufsichtsbehörden in der Union erforderte in Art. 6o bis 76 DSGVO eigene Regelungen zu deren Durchführung. Eine auffällige Veränderung bringt auch Art. 83 DSGVO, der für Verstöße gegen Vorgaben der Verordnung drastische Sanktionen ermöglicht. Nach Art. 83 Abs. 5 DSGVO können bei den dort aufgelisteten Verstößen Geldbußen von bis zu 20 Mio. Euro oder im Fall eines Unternehmens von bis zu $4 \%$ seines gesamten weltweit erzielten Jahresumsatzes des vorangegangenen Geschäftsjahrs verhängt werden, je nachdem, welcher der Beträge höher ist. ${ }^{15}$

12 EG ABI. L 281 vom 23.11.1995, 31.

13 S. Erwägungsgrund 9 DSGVO.

14 Diese Erweiterung sorgt auf dem europäischen Markt für Wettbewerbsgleichheit zwischen Anbietern in der Union und Anbietern außerhalb der Union und vereinfacht die Wahrnehmung von Betroffenenrechten.

15 S. Z.B. BfDI 2016, $18 \mathrm{ff}$. 


\subsection{Das neue Bundesdatenschutzgesetz}

Nationales Datenschutzrecht kann neben der Datenschutz-Grundverordnung mindestens aus drei Gründen anwendbar sein. Es kann erstens eine explizite oder implizite Öffnungsklausel der Datenschutz-Grundverordnung ausfüllen, die den Mitgliedstaaten einen Spielraum einräumt, Regelungen, die die Verordnung nicht enthält, weiterhin anzuwenden oder neu zu erlassen. Es kann weiterhin abstrakte Vorgaben der Datenschutz-Grundverordnung präzisieren und damit Handlungs- und Bewertungsmaßstäbe bieten, die der Verordnung fehlen, sofern sie nicht Entscheidungen der Verordnung widersprechen. Schließlich kann das nationale Datenschutzrecht Vorgaben der Verordnung konkretisieren, die eine unfertige Regelung im Text der Verordnung erst anwendbar machen. Dies ist meist dann der Fall, wenn ursprünglich eine Konkretisierung der Verordnung durch delegierte Rechtsakte oder Durchführungsrechtsakte der Kommission vorgesehen waren, diese Konkretisierungsmöglichkeiten aber entfallen sind. ${ }^{16}$

Die in der Verordnung gegebenen Möglichkeiten hat der deutsche Gesetzgeber inzwischen genutzt und noch in der 18. Legislaturperiode des Deutschen Bundestags ergänzend zur Datenschutz-Grundverordnung mehrere neue Datenschutzgesetze erlassen oder bestehende Gesetze angepasst, darunter ein neues Bundesdatenschutzgesetz und eine Neufassung des Sozialdatenschutzes im Sozialgesetzbuch I und X.

In Art. 1 des Gesetzes zur Anpassung des Datenschutzrechts an die Verordnung (EU) 2016/679 und zur Umsetzung der Richtlinie (EU) 2016/680 (Datenschutz-Anpassungs- und -Umsetzungsgesetz EU - DSAnpUG-EU) vom 30. Juni $2017^{17}$ ist das neue Bundesdatenschutzgesetz enthalten. Dieses tritt mit am 25. Mai 2018 - zusammen mit dem Geltungsbeginn der Datenschutz-Grundverordnung in Deutschland - in Kraft und wird das bisherige Bundesdatenschutzgesetz zu diesem Zeitpunkt vollständig ersetzen. Art. 2 bis 6 DSAnpUG-EU enthalten Anpassungen des Bundesverfassungsschutzgesetzes, des MAD-Gesetzes, des BND-Cesetzes, des Sicherheitsüberprüfungsgesetzes und des Artikel-10-Gesetzes.

Das neue Bundesdatenschutzgesetz regelt nur im zweiten Teil in $\$ \mathbb{\$} 22$ bis 44 allein die Anpassung des allgemeinen Bundesdatenschutzrechts an die Datenschutz-Grundverordnung. Er enthält Vorgaben für besondere Verarbeitungssituationen, für zulässige Zweckänderungen und für Einschränkungen der Rechte der betroffenen Personen. Im dritten Teil finden sich in $\$ \mathbb{S} 45$ bis 84 spezifische Bestimmungen zur Umsetzung der Richtlinie (EU) 2016/680 zum Schutz natürlicher Personen bei der Verarbeitung personenbezogener Daten durch die zuständigen Behörden zum Zwecke der Verhütung, Ermittlung, Aufdeckung oder Verfolgung von Straftaten oder der Strafvollstreckung sowie

16 S. hierzu Roßnagel, in: ders. 2018, § 2 Rn. $15 f f$.

17 BGBI. I, 2097. 
zum freien Datenverkehr und zur Aufhebung des Rahmenbeschlusses 2008/977/ JI des Rates (JI-Richtlinie). ${ }^{18}$ Der ersten Teil schließlich enthält in $\mathbb{s} 1$ bis 21 Regelungen, die sowohl für die Anpassung an die Verordnung als auch der Umsetzung der JI-Richtlinie gelten. Sie betreffen u.a. allgemeine Erlaubnistatbestände sowie die Stellung und die Aufgaben der Bundesbeauftragten für den Datenschutz und die Informationsfreiheit.

Mit dem neuen Bundesdatenschutzgesetz hat der deutsche Gesetzgeber die Öffnungsklauseln der Verordnung - insbesondere in Art. 6, 9, 22 und 23 DSGVO - ausgenutzt und darüber hinaus auch ohne Öffnungsklausel die Regelungen in der Verordnung präzisiert, konkretisiert und ergänzt. ${ }^{19}$ Er hat diese Kompetenzen aber fast ausschließlich dazu benutzt, alte Definitionen beizubehalten, Möglichkeiten zur Verarbeitung personenbezogener Daten zu erweitern und Rechte der betroffenen Personen zu beschränken. ${ }^{20}$

\section{3 Änderungen des SGB I und X}

Eine Neuregelung des Sozialdatenschutzes erfolgte durch Art. 19 und 24 des Gesetzes zur Änderung des Bundesversorgungsgesetzes und anderer Vorschriften vom 24. Juli 2017..$^{21}$ Der Gesetzgeber stützt sie durchgängig - ohne weitere Differenzierung - auf die Öffnungsklauseln für den öffentlichen Bereich in Art. 6 Abs. 2 und 3 sowie auf die Öffnungsklausel zur Verarbeitung besonderer Kategorien von personenbezogenen Daten in Art. 9 Abs. 2 DSGVO. ${ }^{22}$ Neugefasst wurden die Regelung des Sozialgeheimnisses in $\mathbb{} 35$ SCB I, die Definitionen

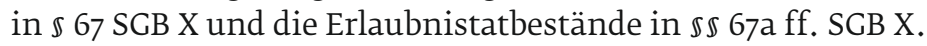

Hinsichtlich des Verhältnisses der Neuregelungen des Sozialdatenschutzes zur Datenschutz-Grundverordnung ist die amtliche Begründung widersprüchlich. Einerseits geht sie davon aus, dass „die datenschutzrechtlichen Regelungen des Sozialdatenschutzes ... das bereichsspezifische Datenschutzrecht abschließend regeln“. Andererseits nimmt sie an, dass die Verordnung unmittelbare Geltung habe und künftig unmittelbar neben den Regelungen zum Sozialdatenschutz anzuwenden sei. ${ }^{23}$ Die Öffnungsklauseln des Art. 6 Abs. 2 und 3 DSGVO sollen jedoch gerade dazu dienen, gewachsene hochkomplexe Datenschutzregelungen, wie sie für die Systeme der sozialen Sicherheit bestehen, die spezifische Eigenheiten der Mitgliedstaaten betreffen, beibehalten zu können. ${ }^{24}$ Daher kann auch der deutsche Regelungskomplex des Sozialdatenschutzes als ein in sich abgeschlossenes System beibehalten werden.

18 Richtlinie (EU) 2016/680, EU ABI. 119 vom 4.5.2016, 89.

19 S. hierzu auch Roßnagel, DuD 2017, 277; Geminn, DuD 2017, 295 (297f.); Johannes/Richter, DuD 2017, $300 f f$.

20 S. Greve, NVwZ 2017, 737; Kühling, NJW 2017, 1985; Roßnagel, DuD 2017, 277 (281).

21 BGBl. I, 2541.

22 Amtliche Begründung, BT-Drs. 18/12611, 104f., $110 f f$.

23 BT-Drs. 18/12611, 105.

24 S. hierzu Roßnagel, DuD 2017, 290 (291f.). 


\section{4 Änderungen des $\oint 203$ StGB}

Unabhängig von der Datenschutz-Grundverordnung hat das Gesetz zur Neuregelung des Schutzes von Geheimnissen bei der Mitwirkung Dritter an der Berufsausübung schweigepflichtiger Personen vom 30. Oktober $2017^{25}$ in Art. 1 das Strafgesetzbuch geändert. Die wichtigste Änderung ist die Aufhebung von

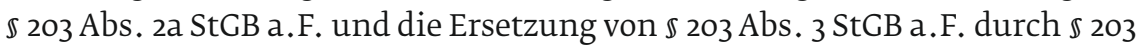
Abs. 3 und 4 StGB n.F. Damit zielt der Gesetzgeber darauf ab, „die Möglichkeiten für Berufsgeheimnisträger zu erweitern, sich im Rahmen ihrer beruflichen oder dienstlichen Tätigkeit ohne (straf-)rechtliches Risiko der Mitwirkung dritter Personen zu bedienen“. ${ }^{26}$

Ergänzend nimmt das Gesetz Änderungen in der Strafprozessordnung in Art. 2, der Bundesrechtsanwaltsordnung in Art. 3, der Bundesnotarordnung in Art. 4, der Patentanwaltsordnung in Art. 5, im Steuerberatungsgesetz in Art. 8 und in der Wirtschaftsprüferordnung in Art. 9 und in vielen weiteren Gesetzen als Folgeänderungen in Art. 10 vor. Diese weiteren Artikel enthalten überwiegend Regelungen zur Inanspruchnahme von Dienstleistungen. Die teilweise schon satzungsrechtlich bestehende Pflicht für bestimmte Berufsgeheimnisträger, ihre Mitarbeiter zur Verschwiegenheit zu verpflichten, wird in die entsprechenden Gesetze überführt. 